\title{
Nouvelle approche de caractérisation des flux de patients
}

\author{
Jessica Pinaire ${ }^{1,2}$, Jérôme Azé ${ }^{2}$, Sandra Bringay ${ }^{2,3}$, \\ Pascal Poncelet $^{2}$, Christophe Genolini ${ }^{4,5}$, Paul Landais ${ }^{1}$ \\ 1. EA 2415, IURC, Université Montpellier \\ 641, avenue du doyen Gaston GIRAUD 34093 Montpellier cedex 5, France \\ prenom.nom@umontpellier.fr \\ 2. Université Montpellier, LIRMM UMR 5506, CC477 \\ 860 rue St Priest, 34095 Montpellier Cedex 5, France \\ prenom.nom@lirmm.fr \\ 3. AMIS, Université Paul-Valéry Montpellier 3 \\ Route de Mende, 34199 Montpellier Cedex 5, France \\ sandra.bringay@univ-montp3.fr \\ 4. CeRSM (EA 2931), Université Paris Nanterre \\ 200 avenue de la république, 92000 Nanterre, France \\ christophe.genolini@parisnanterre.fr \\ 5. Start'up Zebrys, 5 place Jean Deschamps, 31100 Toulouse, France
}

\begin{abstract}
RÉSUMÉ. Mieux connaître les trajectoires hospitalières des patients est essentiel pour planifier les soins. À partir des données administratives, nous extrayons des motifs spatio-temporels et les utilisons pour identifier des profils de délais inter-séjours et d'évolution de tarifs des parcours de soins. Cette approche pourrait favoriser l'amélioration des stratégies de soins.

ABSTRACT. A better knowledge of patient hospital flows can be decisive for health planning. We extracted spatio-temporal patterns from the French hospital Prospective Payment System, and used them to identify profiles of care pathways times and costs. This approach may support renewed care management strategies.

MOTS-CLÉS : flux de patients, données médico-économiques, infarctus du myocarde, motifs spatiotemporels, classification de données longitudinales.

KEYWORDS: patient flows, prospective payment system, myocardial infarction, spatio-temporal patterns, longitudinal data clustering.
\end{abstract}

DOI:10.3166/tsi37-0003 @ 2018 Lavoisier

Technique et science informatique $-\mathrm{n}^{\circ} 1-6 / 2018,65-81$ 


\section{Introduction}

La planification sanitaire vise à assurer l'efficience du système de santé mais aussi à réduire les inégalités et les disparités géographiques d'accès aux soins. Une manière efficace de contribuer à améliorer la planification sanitaire est d'avoir une meilleure compréhension des trajectoires de soins considérées comme un indicateur des besoins réels des patients. En effet, ces trajectoires sont une source d'informations sur la quantité et le type de soins, mais également sur les délais et les coûts de prise en charge. Or, ces éléments sont essentiels pour la gestion des soins hospitaliers et constituent de véritables enjeux dans l'amélioration de la qualité des soins et la réduction de leurs coûts $(?, ?)$.

Les bases nationales de données médico-économiques, issues du Programme de Médicalisation du Système d'Information (PMSI), constituent une importante source d'informations sur l'hospitalisation en France (?, ?). Malgré leurs limites en termes de couverture, de qualité et de validité, ces données peuvent avoir d'autres finalités que la facturation telles que l'amélioration de la prise en charge des patients $(?, ?)$, la prédiction de l'évolution des soins (?, ?) ou encore la planification de leurs coûts (?, ?). Un défi associé à ces données est de concevoir des outils permettant à la fois de traiter une telle masse de données et d'en extraire une information pertinente. À l'ère de la numérisation et de la modernisation des systèmes de santé ${ }^{1}$ de nombreux chercheurs se sont intéressés aux méthodes issues de la fouille de données et ont montré leur efficacité dans le domaine de la santé $(?, ?)$.

Dans cet article, nous proposons une approche innovante pour caractériser les flux de patients, illustrée par l'hospitalisation pour infarctus du myocarde (IM). À partir des données du PMSI, nous avons extrait des motifs caractérisant des trajectoires en adaptant une méthode de recherche de motifs spatio-temporels (?, ?). L'originalité ici est de ne plus considérer la proximité géographique des objets, mais la proximité des pathologies codées dans les bases du PMSI. Les motifs spatio-temporels ont été intégrés dans un outil de visualisation et permettent de reconstituer les différentes évolutions possibles de la maladie étudiée, l'IM. Par ailleurs, nous avons groupé ces trajectoires pour identifier des tendances dans les délais inter-séjours et l'évolution des tarifs. L'association de ces profils de délais et de tarifs permet d'envisager une aide pour la mise en œuvre de nouvelles stratégies d'organisation des soins.

Dans la section 2, nous nous focalisons sur le contexte plus spécifique de l'IM et nous posons une hypothèse forte sur l'intérêt à la fois des données médicoéconomiques et des motifs que nous extrayons comme base de nos travaux. Ensuite, dans la section 3, nous introduisons les différentes techniques utilisées et présentons notre processus de caractérisation des trajectoires de patients. Dans la section 4, nous identifions à la fois des phénomènes de groupes, des profils d'évolution de tarifs et d'occurrence des hospitalisations chez les patients de +65 ans. Enfin, nous discutons des résultats obtenus et des limites de cette approche dans la section 5.

1. Notamment en France avec la loi 2016-41 de modernisation du système de santé. 


\section{Contexte}

\subsection{Motivations}

L'Organisation Mondiale de la Santé (OMS) recense 32,4 millions de cas d'IM et d'accidents vasculaires cérébraux par an $(?, ?)$. De manière générale, l'OMS prévoit une augmentation du fardeau des maladies cardiovasculaires à hauteur de 11,06\% d'ici 2030, portant le nombre de cas à environ 36,2 millions (?, ?). Par ailleurs, les personnes présentant un IM ont un risque de récidive et/ou de développement d'affections liées à l'insuffisance coronarienne six fois plus élevé que celles n'ayant pas d'antécédent d'IM $(?$, ?). De plus, la part des décès dus aux maladies cardiovasculaires représente approximativement $31 \%$ de l'ensemble des décès dans le monde : c'est la première cause de mortalité dans le monde (?, ?). Ainsi, l'IM constitue-t-il un enjeu majeur de santé publique, induisant une forte consommation des ressources en santé. Dans un tel contexte, outre la lutte contre les facteurs de risques modifiables, l'amélioration de la planification sanitaire constitue un axe d'exploration complémentaire important. C'est la raison pour laquelle nous avons souhaité mieux appréhender cette pathologie par la caractérisation des flux de patients hospitalisés pour IM en France.

Les complications liées à l'IM sont déjà bien décrites (?, ?), en revanche, il n'est pas précisé dans quelles proportions les réadmissions se répartissent selon ces différentes complications, ni à quel moment du parcours elles surviennent et dans quels délais. Dans une situation de réduction des coûts de santé, il est important de caractériser ces flux de patients selon ce critère. Toutefois, la plupart des méthodes qui sont mises en oeuvre pour l'étude des coûts déterminent un coût cumulé moyen prenant en compte l'ensemble des prises en charge d'un individu donné : prise en charge en urgence, hospitalisation, médicaments, suivi en ville... L'étude de ces coûts moyens (?, ?; ?) ne permet pas de discerner la part de l'augmentation du nombre de cas à traiter du coût de la prise en charge individuelle (nouvelles technologies interventionnelles par exemple). Pourtant, cette information pourrait avoir un impact sur le choix de la stratégie de soins.

\subsection{Hypothèses}

L'IM est une pathologie grave nécessitant une hospitalisation et une prise en charge dans une unité de soins intensifs de cardiologie. Or, la base nationale du PMSI collige l'ensemble des séjours hospitaliers sur tout le territoire français pour l'ensemble des établissements de soins dans un recueil exhaustif et systématique. De plus, grâce à l'identifiant anonyme de patient, il est possible de retracer le parcours d'un patient sur tout le territoire national quel que soit l'établissement de soins fréquenté (?, ?). Ces données ont servi pour la création d'algorithmes de repérage des pathologies à partir des données médico-économiques (?, ?), pour la recherche d'associations de maladies $(?, ?)$, pour évaluer l'impact économique de prise en charge du patient $(?, ?)$ ou encore pour identifier des facteurs prédictifs de ré-hospitalisation $(?, ?)$. Elles ont aussi servi dans la construction de score prédictif du décès à l'issue d'une chirurgie (?, ?). Étant 
donné les divers exemples d'utilisation de ces données, nous supposons que mener nos investigations à partir de ces bases de données est un choix adéquat. À partir, de ces données, nous pourrons reconstituer les parcours de soins des patients caractérisés par leur séquence de motifs d'hospitalisation. Pour le PMSI, le diagnostic principal (DP). est le diagnostic qui a motivé l'hospitalisation.

Dans un objectif d'utilité prévisionnelle dans l'organisation sanitaire, nous nous sommes intéressés aux parcours communs les plus fréquents. La recherche de phénomènes communs dans les parcours de soins est analogue à la détection de phénomènes de groupes pour les objets mobiles (?, ?). Ces motifs ont été utilisés par exemple, pour suivre des trajectoires de migrations d'oiseaux (?, ?), ou encore des saumons (?, ?). Ils ont également été utilisés dans d'autres domaines tels que la régulation du trafic routier $(?, ?)$ ou encore l'adaptation des infrastructures pour faciliter les déplacements des riverains (?, ?). De manière analogue aux objets mobiles, un parcours de soins est une succession chronologique d'évènements survenant à des estampilles de temps différentes. Ainsi, en redéfinissant la notion de spatialité par le type de séjour hospitalier et la notion de temps par l'occurrence d'un évènement, nous pouvons assimiler une trajectoire de patient à une trajectoire d'objet mobile. Par conséquent, pour caractériser les flux de patients, nous faisons l'hypothèse que l'extraction de motifs spatio-temporels est pertinente pour la détection de parcours communs.

Enfin, pour caractériser ces flux selon des critères de délais et de coûts, nous envisageons de déterminer des profils d'occurrence et de tarifs ${ }^{2}$ de ces évènements. Or, identifier des profils revient à regrouper dans une même catégorie des évolutions similaires. Les trajectoires de délais et de tarifs étant des données longitudinales, cela revient à identifier des courbes qui évoluent de la même façon ou encore qui ont la même forme. À cet effet, nous utilisons une méthode de classification de données longitudinales selon la forme des courbes (?, ?). Cette méthode a, par exemple, été utilisée pour établir un lien entre l'évolution de l'ADN et la production chez les vaches laitières (?, ?). Elle a également été appliquée pour la description des différents profils dans la perte des facultés d'une personne atteinte de la maladie d'Alzheimer (?, ?). De plus, elle a favorisé l'identification des différents profils hormonaux dans le cycle menstruel à partir des mesures de l'hormone LH chez des femmes fertiles (?, ?). Nous partons de l'hypothèse que ce type de méthode est adapté pour identifier des profils dans les trajectoires de délais et de tarifs.

2. En effet, dans les bases PMSI, nous n'avons pas accès aux coûts réels mais uniquement aux tarifs des séjours. 


\section{Méthodes}

\subsection{Définitions préliminaires}

\subsubsection{Motifs spatio-temporels}

La fouille de données spatio-temporelles consiste à discerner des ensembles d'objets restant groupés spatialement sur une même période. Il existe de nombreux motifs spatio-temporels. Nous présentons uniquement ceux utilisés dans cet article.

De manière intuitive, un essaim (?, ?) est un groupe d'au moins mino objets mobiles prenant la même valeur pour au moins mint estampilles de temps. De manière plus formelle, un essaim peut être défini de la façon suivante:

On note $\mathrm{V}$ un ensemble d'états possibles (e.g. "Douleur thoracique" ; "Diabète"...) $\mathrm{T}=\left\{t_{1}, \ldots, t_{p}\right\}$ un ensemble d'estampilles temporelles; $\mathrm{O}$ un groupe de $n$ objets mobiles à valeur dans $\mathrm{V}$ (par exemple, des individus ayant une maladie); on note $O_{t}^{i} \in \mathrm{V}$ l'état de l'individu $j$ au temps $t$. Lorsque plusieurs individus ont la même valeur aux mêmes estampilles de temps, on dit qu'ils sont dans le même cluster. On cherche à identifier un groupe d'objets qui se trouveraient dans le même cluster pendant une certaine durée : soit mino un support minimum, donné par l'utilisateur, correspondant au nombre d'objets minimum devant être ensembles ; mint, le nombre minimum d'estampilles temporelles pendant lesquelles au moins mino objets de sont regroupés.

DÉFINITION 1 (Essaim). - Étant donné un ensemble d'objets mobiles $\mathrm{O}$ et des estampilles temporelles $\mathrm{T}$, un support minimum mino et un nombre minimum d'estampilles temporelles mint, un essaim (ou swarm en anglais) est un couple $(O, T) \in$ $(\mathrm{O}, \mathrm{T})$ vérifiant :

1) À chaque estampille temporelle de T, il y a au moins un cluster contenant tout objet de $O: \forall t_{i} \in T, \exists$ ctel que $O \subseteq$ coù cest un cluster ;

2) Il y a au moins mino objets : $|O| \geq$ mino;

3) Il y a au moins mint estampilles temporelles : $|T| \geq$ mint.

Un essaim clos est un essaim dont il n'est pas possible d'extraire un essaim plus petit. Cependant si $(O, T)$ est un essaim, tous les couples $\left(O^{\lrcorner}, T^{\lrcorner}\right)$tels que $O^{\lrcorner} \subseteq O$ et $T^{\jmath} \subseteq T$ peuvent aussi être des essaims et l'information obtenue est donc redondante. La notion d'essaim clos introduite dans (?, ?) permet d'éviter de rechercher les essaims redondants :

DÉFINITION 2 (Essaim clos). - Un couple (O, T) est un essaim clos (closed swarm) $s$ 'il vérifie :

1) $(O, T)$ est un essaim;

2) $(O, T)$ est objet-fermé : $\$ O^{\top}$ tel que $(O, T)$ est un essaim et $O \subset O^{\top}$;

3) $(O, T)$ est temporel-fermé $: \$ T$ tel que $(O, T)$ est un essaim et $T \subset T$. 
EXEMPLE 3. - Pour illustrer ces définitions, considérons les évènements de quatre patients. Ici, le temps est divisé en estampilles temporelles correspondant à la survenue d'une hospitalisation. La figure 1 décrit les différents (DP) correspondant aux motifs d'hospitalisation (I21 : IM aigu; R07 : Douleurs thoraciques; E14 : Diabète; I20 : Angine de poitrine; I70 : Athérosclérose) de ces patients.

$\mathrm{Si} \min _{0}=2$ et $\min _{t}=2$, nous identifions les essaims suivants : $\left(\left\{P_{1}, P_{3}\right\}\left\{0, t_{1}\right)\right\}\left(B_{1}, P_{3},\right\}\left\{1, t_{2}\right\}$ et $\left.\left(P_{1}, P_{3}\right\}\left\{t_{2}, t_{3}\right\}\right)$. Les patients $P_{1}, P_{3}$ ont le même DP I21 en to, et le même DP E14 en t1. Ils ont également le même DP I20, en $t_{2}$ et aussi en $t_{3}$ I70. Notons que, ces groupes sont redondants et peuvent être réunis dans l'essaim $\left(\left\{P_{1}, P_{3}\right\},\left\{t_{0}, t_{1}, t_{2}, t_{3}\right\}\right)$ qui est un essaim clos.

\begin{tabular}{lllll}
\hline & \multicolumn{4}{c}{ Estampilles de temps } \\
2-5 Patient & $t_{0}$ & $t_{1}$ & $t_{2}$ & $t_{3}$ \\
\hline$P_{1}$ & I21 & E14 & I20 & I70 \\
$P_{2}$ & I21 & R07 & I20 & \\
$P_{3}$ & I21 & E14 & I20 & I70 \\
$P_{4}$ & I21 & R07 & I20 & \\
\hline
\end{tabular}

Figure 1. Trajectoires de patients. Le temps est lié à la survenue d'une hospitalisation (de gauche à droite la visualisation des trajectoires et la base séquentielle).

\subsubsection{Classification de données longitudinales quantitatives}

Les données longitudinales sont des données pour lesquelles chaque variable a été mesurée plusieurs fois au cours du temps pour un même individu. Une manière d'analyser ces données est de les partitionner avec des méthodes comme celle des kmoyennes ou encore des variantes de cette méthode (?, ?). Dans cet article, nous nous intéressons à l'évolution d'un phénomène plutôt qu'à son moment d'apparition. De ce fait, nous avons choisi la méthode kmlShape qui prend en compte la forme des courbes (?, ?). Dans la suite, nous présentons son principe de fonctionnement et nous définissons les deux concepts clés de distance et de moyenne sur lesquels s'appuie cette méthode.

La classification par les k-moyennes est un algorithme de partitionnement utilisé pour les données longitudinales. Elle alterne deux étapes : 1) le calcul des trajectoires moyennes de chaque groupe ; 2) le calcul des distances entre individus et la moyenne de son groupe. Cet algorithme affecte un individu au groupe dont il est le plus proche. L'algorithme kmlShape (?, ?) repose sur ces deux étapes en remplaçant la moyenne et la distance classique par la moyenne de Fréchet pour le calcul des trajectoires moyennes et la distance de Fréchet pour le calcul des distances entre individus et représentants de classe. Ces mesures confèrent à kmlShape la capacité de respecter la forme des courbes, ce qui est nécessaire car les durées entre évènements peuvent beaucoup varier d'un patient à l'autre. 
Plus formellement, la notion de distance de Fréchet nécessite l'introduction du concept de reparamétrisation :

DÉFINITION 4 (Reparamétrisation). - Soit $t \in \mathrm{R}$ un réel et $[0, t]$ un intervalle de $\mathrm{R}$. Une reparamétrisation de $[0, t]$ est une fonction continue, croissante, surjective de $[0, t] \rightarrow[0, t]$.

Nous introduisons quelques notations pour les définitions de distances : $d$ la distance euclidienne ; $P$ et $Q$, deux courbes de $[0, t]$ dans $\mathrm{R}$; A l'ensemble de toutes les reparamétrisations de $[0, t]$; a et $\beta \in \mathrm{A}$ deux reparamétrisations et $s \in[0, t]$ un réel.

Par analogie, une courbe $P$ peut-être assimilée à la trajectoire d'un mobile voyageant à vitesse constante et $P_{\curvearrowright} a$ est la même trajectoire que $\mathrm{P}$ mais parcourue par un autre mobile avec une vitesse $a$.

DÉFINITION 5 (Distance de Fréchet). - La distance entre deux courbes $P$ et $Q$ reparamétrées par a et $\beta$ au temps s est :

$$
\left.d_{a, \beta, s}(P, Q)=d(P \circ a(s), Q \circ \beta(s))=d^{\cdots} \begin{array}{c}
a(s) \\
P(a(s))
\end{array}\right)^{\Sigma} \quad \begin{aligned}
& \beta(s) \\
& Q \Sigma(s))
\end{aligned}
$$

La distance entre deux courbes $P$ et $Q$ reparamétrées par a et $\beta$ est la distance maximale des distances $d_{a, \beta, s}(P, Q)$ avec $s$ variant dans $[0, t]$ :

$$
d_{a, \beta}(P, Q)=\operatorname{Max}_{s \in[0, t]}\left(d_{a, \beta, s}(P, Q)\right)
$$

Alors, la distance de Fréchet entre $P$ et $Q$ est le plus petit maximum possible entre $P$ et $Q$ après reparamétrisation de $P$ et de $Q$ :

$$
\operatorname{DistFrechet}(P, Q)=\operatorname{Inf}(a, \beta) \in \mathrm{Az} d d_{a, \beta}(P, Q)
$$

Si la variable d'intérêt est mesurée avec des échelles de temps différentes cela peut impacter le calcul de distance. Par voie de conséquence, cela peut affecter le résultat du partitionnement. Un exemple illustre ce propos dans la figure 2. Dans le schéma de gauche, selon la distance de Fréchet, la courbe Q est plus proche de la courbe R que de $\mathrm{P}$. Tandis que, dans le schéma de droite, la courbe $\mathrm{Q}$ est plus proche de la courbe $\mathrm{P}$ que de R. La définition qui suit permet de prendre en compte les changements d'échelle.

Figure 2. Impact du changement d'échelle (paramètre lambda) dans le calcul de la distance de Fréchet.

DÉFINITION 6 (Distance de Fréchet généralisée). - Soit $\lambda$ le paramètre de l'échelle de temps. La distance de Fréchet généralisée au paramètre $\lambda$ entre deux courbes $P$ et $Q$ est la distance de Fréchet obtenue après la transformation affine $A: \mathrm{R}^{2} \rightarrow \rightarrow \mathrm{R}^{2}$

$$
(x, y) \rightarrow(\lambda x, y)
$$

c'est-à-dire DistFrechet $(P, Q)=\operatorname{Inf}(a, \beta) \in A z \operatorname{Max}(P \circ a \circ A, Q \circ \beta \circ A)$ 
DÉFINITION 7 (Moyenne de Fréchet). — La moyenne de Fréchet entre deux courbes $P$ et $Q$ est définie par :

$$
\operatorname{MeanFrechet}_{\lambda}(P, Q)=\frac{-P \circ Q \circ A+Q \circ \beta \circ A}{2}
$$

Cette définition peut se généraliser à $n$ trajectoires chacune de $\mathrm{t}$ points ${ }^{3}$ en procédant à des regroupements deux à deux. Dans une population de $n$ individus, il est possible de constituer des paires d'individus (avec un poids égal à 1) et de calculer les moyennes de Fréchet pour chaque paire. Les moyennes ainsi déterminées peuvent être à leur tour combinées par paires. Il est alors possible de calculer les moyennes de Fréchet pour chaque paire en les pondérant par le nombre d'individus utilisés pour le calcul de chacune. Ce principe est réitéré jusqu'à obtenir une moyenne unique. Cette méthode de calcul pas à pas réduit la complexité de l'algorithme de $O\left(t^{n}\right)$ à $O\left(n t^{2}\right)$.

EXEMPLE 8. - La figure 3 présente un exemple de calcul de distances moyennes, selon la méthode de Fréchet ou la méthode euclidienne, entre une série de courbes (dessinées en gris). Dans cet exemple, nous pouvons observer que la moyenne de Fréchet (courbe orange et trait épais) conserve la forme générale des courbes contrairement à la distance euclidienne (courbe noire et trait épais).

Figure 3. Exemple de comparaison entre la moyenne de Fréchet et la distance euclidienne.

\subsection{Protocole de caractérisation des flux de patients}

Notre protocole de caractérisation des flux de patients se décompose en trois grandes étapes. La première étape est la constitution de la base séquentielle à partir des données du PMSI. Ensuite, la deuxième étape consiste à extraire puis à trier des motifs spatio-temporels à partir de la base séquentielle dans le but de caractériser les schémas de flux de patients. Pour finir, la troisième étape consiste à classer les trajectoires de délais inter-séjours et de tarifs de ces évènements dans le but d'identifier des profils de délais et d'évolution de tarifs. Ces étapes sont détaillées dans la suite de cette section et sont schématisées dans la figure 4 .

Figure 4. Schéma du protocole de caractérisation des flux de patients.

3. Dans les définitions de la reparamétrisation et de la distance de Fréchet, nous avons considéré t réel. Dans la pratique médicale, t est un entier. 


\subsection{1. Étape 1. Acquisition des données}

Cette première partie de constitution de la base séquentielle se réalise comme suit :

a. À partir de la base nationale du PMSI, sur une période recouvrant six années allant de 2009 à 2014, nous sélectionnons les patients ayant eu un séjour comprenant un DP ou motif d'hospitalisation d'IM et/ou un acte de cardiologie interventionnelle ${ }^{4}$ au cours de cette période.

b. Nous construisons la base séquentielle en nous intéressant aux DP. Pour chaque patient, nous récupérons l'ensemble de ses séjours sur la période 2009 à 2014, excepté les séjours pour séances (radiothérapie, dialyses, chimiothérapie, etc.) et les prestations inter-établissements : lorsque l'on transfère un patient dans un autre établissement pour lui faire faire un acte (e.g. une coronarographie) car le premier n'a pas l'équipement nécessaire pour le réaliser. Ainsi, chaque patient a une séquence de $\mathrm{DP}$, de longueur égale à son nombre de séjours.

c. Nous procédons à des prétraitements de ces séquences : 1) filtrage des évènements en ne retenant que ceux liés à l'affection cardiaque ; 2) recalage des séquences selon un temps relatif correspondant à la survenue d'un évènement hospitalier. Les séquences de DP ainsi triées constituent la trajectoire du patient. Elles seront ensuite analysées lors de l'étape 2.

d. Enfin, nous préparons les données longitudinales quantitatives : 1) le délai interséjours correspond au nombre de jours écoulés entre le dernier jour d'une hospitalisation et le premier jour de l'hospitalisation suivante ; 2) le tarif d'un séjour a été déterminé en tenant compte de la valorisation du séjour liée au Groupe Homogène de Séjour (GHS) et des suppléments ${ }^{5}$. Bien que cette valorisation ne soit pas un calcul exact du tarif de chacun des séjours, elle fournit une bonne estimation du tarif réel de l'hospitalisation. Ces données seront ensuite analysées lors de l'étape 3.

\subsubsection{Etape 2. Extraction de motifs spatio-temporels}

Le processus de fouille, s'effectue de la façon suivante :

a. Nous extrayons des essaims clos à l'aide de l'algorithme Get_Move (?, ?) dans la base séquentielle des trajectoires de patients. La fouille génère de nombreux motifs qui sont filtrés en ne retenant que ceux qui concernent au moins $1 \%$ des patients du contexte étudié.

b. Nous représentons les parcours de patients, identifiés dans l'étape a, à l'aide d'un diagramme de Sankey ${ }^{6}: 1$ ) les sommets sont rassemblés selon une cohérence médicale $(?, ?) ; 2)$ des groupes de flux sont créés selon le premier évènement de la trajectoire.

4. Nous avons retenus les actes effectués lors d'un IM comme par exemple le stenting ou l'angioplastie.

5. Les suppléments pour la réanimation, les soins intensifs et les soins continus.

6. Il s'agit d'un diagramme de flux dans lequel la largeur des bandes est proportionnelle au nombre de patients considérés. 


\subsection{3. Étape 3. Identification de profils d'occurrence et d'évolution de tarifs}

Le processus de classification comprend trois étapes :

a. Nous avons noté de fortes disparités de tarifs entre les trajectoires. Nous avons alors, appliqué une transformation à l'aide du logarithme népérien afin de lisser les courbes et faciliter leur classification ${ }^{7}$. Dans la suite, ces deux types de trajectoires : délais et tarifs sont traités de façon séparée.

b. Nous classons les trajectoires de délais puis celles de tarifs à l'aide de la méthode kmlShape (?, ?). Pour déterminer le nombre "optimal" de classes, nous avons opté pour une méthode analytique ${ }^{8}$. Cette dernière consiste à utiliser la répartition de classes, issue de la classification par kmlShape, dans un modèle prédictif du décès. La répartition offrant les meilleurs résultats est celle qui sera retenue. Plus concrètement, soit $k$ le nombre de classes, nous classons les trajectoires en faisant varier $k$ entre 2 (afin d'obtenir au moins deux classes) et 6 (au-delà, les classes ne seraient pas interprétables). À chaque itération, nous modélisons l'état final du patient (vivant/décédé) par une régression logistique en utilisant le découpage obtenu par kmlShape comme prédicteur. Nous définissons le $k$ "optimal" comme étant celui pour lequel le modèle est le meilleur (critères de décision: critère d'information d'Akaiké (AIC) et valeur de p). In fine, chaque patient est affecté à une classe dont le représentant est la moyenne de Fréchet.

c. Puis, nous établissons la répartition des groupes de patients, de l'étape 2, dans les classes de délais et de tarifs ainsi obtenues.

\section{Expérimentations}

L'incidence de l'IM est plus importante chez les hommes, même si la différence entre les hommes et les femmes s'atténue avec l'âge. Le risque de développement d'un IM est généralement plus tardif chez les femmes, en moyenne une dizaine d'années plus tard que chez les hommes (?, ?). Bien que l'IM affecte moins les femmes, leur taux de mortalité par IM est plus élevé que celui des hommes (?, ?). Ainsi, pour ces raisons, nous avons analysé les flux de patients en distinguant les hommes des femmes. Nous présentons les résultats pour des patients âgés de +65 ans.

\subsection{Identification des schémas de flux}

Dans la figure 5 (a), nous identifions chez les femmes de +65 ans trois flux de patients dont les évènements initiaux sont respectivement Angine de poitrine, Cardiopathie ischémique et IM. Les flux se séparent ensuite en plusieurs branches dont les évènements suivants sont ceux cités précédemment, auxquels s'ajoute le Décès. À partir de la troisième hospitalisation apparaissent deux nouveaux évènements : les

7. Pour éviter d'obtenir un trop grand nombre de classes ce qui deviendrait ardu à interpréter.

8. En effet, kmlShape ne se basant pas sur des distances classiques, nous ne pouvons pas utiliser les indicateurs classiques de la littérature. 
Troubles du rythme et l'Insuffisance cardiaque. Nous remarquons, par ailleurs, que les flux de patientes sont de plus en plus réduits au fur à mesure du temps.

Les schémas de flux chez les hommes sont plus complexes. La figure 5 (b) montre un diagramme avec une estampille de temps supplémentaire et une plus grande diversité des sommets à chaque étape. Toutefois, la majeure partie des patients évolue dans des schémas de flux similaires à ceux décrits dans le cas des femmes. Intéressons-nous à ce qui les différencie. Parmi les évènements initiaux, il y a en plus les évènements suivants : Greffe cardiaque, Troubles du rythme, Autres complications, Insuffisance cardiaque et Observation surveillance et suivi de traitement. Ces évènements apparaissent jusqu'à la quatrième estampille de temps à quelques exceptions près. Les flux initialisés par ces évènements se divisent ensuite entre Cardiopathie ischémique et Angine de poitrine. La suite du parcours est identique à celle déjà décrite pour les femmes. Une autre particularité chez les hommes est l'évènement Anévrisme présent à la troisième estampille de temps.

\title{
(a) Hommes +65 ans
}

\section{(b) Femmes +65 ans}

\author{
Légende \\ A.P : Angine de poitrine ACO : Autres complications \\ ANV : Anévrisme $\quad$ AUT : Autres troubles \\ DC : Décès $\quad$ GRF : Greffes cardiaques - \\ TSC : Ischémie
TTT : Observation, surveillance et suivi de traitement - Résultats anormaux d'examens \\ Figure 5. Flux de patients de +65 ans dans les trajectoires de DP dans un \\ diagramme de Sankey.
}

\subsection{Identification des profils de délais et de tarifs}

\subsubsection{Le cas des femmes}

La classification des trajectoires de délais donne trois classes (voir figure 6 (a)). Ces classes sont des courbes différenciées par les couleurs et l'épaisseur du trait : bleue et trait épais (classe $B$ ), magenta et trait fin (classe $M$ ) et verte avec trait très épais (classe $V$ ). Elles caractérisent des profils différents de délais de ré-hospitalisations. En effet, nous observons dans la figure $6(\mathrm{a})$, que les courbes ont des formes très différentes ${ }^{9}$. La classe $B$ représente 3070 patientes ayant des délais courts (inférieurs à quatre mois) qui augmentent puis diminuent. La classe $M$ concerne 1480 patientes ayant des évènements espacés en début de parcours puis plus rapprochés.

9. Le graphique se lit de la façon suivante, en $t_{0}$ est affiché le temps qui sépare la première hospitalisation de la seconde. Par exemple, la classe $M$, représentée par la courbe magenta (trait fin), a une ordonnée égale à 500 en $t_{0}$ : le délai entre la première hospitalisation et la seconde, pour les femmes de cette classe, est de 500 jours. 
La classe $V$ caractérise 930 patientes ayant des délais interhospitalisations qui augmentent constamment donc des hospitalisations de plus en plus espacées dans le temps. La répartition des groupes de flux dans les classes de délais (voir figure 6 (c)) montre qu'une majorité de la population a des délais qui diminuent en fin de parcours.

La classification des trajectoires de tarifs donne également trois classes équiréparties (voir figure 6 (b)). La classe $B$ représente 1910 patientes ayant des séjours avec des tarifs augmentant au fur à mesure du parcours et diminuant légèrement en fin de parcours. La classe $M$ caractérise 1840 patientes ayant des séjours avec des tarifs en forte diminution, puis ré-augmentant fortement en fin de parcours. Pour finir, la classe $V$ caractérise 1730 patientes avec des tarifs de séjours qui augmentent en début de parcours puis diminuent. La répartition des groupes de flux dans les classes de tarifs (figure 6 (c)) montre, dans le cas du groupe $I M$, qu'il y a une majorité de profils avec des tarifs élevés en début de parcours mais qui diminuentensuite.

(a)

\begin{tabular}{cr|ccc|ccc}
\hline Groupe & & \multicolumn{3}{|c|}{ Classes de délais } & \multicolumn{3}{c}{ Classes de tarifs } \\
Nom* & Effectif & $B$ & $M$ & $V$ & $B$ & $M$ & $V$ \\
\hline Angine de poitrine & 1711 & 47 & 28 & 25 & 41 & 23 & 36 \\
Cardiopathie ischémique & 1201 & 52 & 32 & 16 & 52 & 19 & 29 \\
$I M$ & 2651 & 45 & 34 & 21 & 25 & 36 & 39 \\
\hline *Le nom du groupe correspond au premier évènement du parcours de soins.
\end{tabular}

(c)

Figure 6. Profils de délais et de tarifs pour les femmes de +65 ans. Classes de (a) délais et (b) tarifs obtenues par kmlShape. (c) Répartition (en pourcentages) des groupes de flux dans les classes de délais et de tarifs $(B, M, V$ correspondent aux clusters des graphes (a) et (b)).

\subsubsection{Le cas des hommes}

Comme chez les femmes, la classification des trajectoires de délais donne trois classes (voir figure 7 (a)). Les profils sont relativement similaires à ceux identifiés chez les femmes à un groupe près. La classe $B$ représente 9737 patients ayant des délais de plus en plus courts. La classe $M$ concerne 2696 patients ayant des évènements de plus en plus espacés allant de quatre à cinq mois jusqu'à plus de deux ans. La classe $V$ caractérise 2546 patients ayant des délais courts en début de parcours, un évènement très éloigné en milieu de parcours et des délais courts à nouveau. De même que chez les femmes, la répartition des groupes de flux dans les classes de délais (voir figure 7 (c)) montre qu'une majorité de la population a des délais de plus en plus réduits.

Contrairement au cas des délais, les profils de tarifs mis en évidence chez les hommes sont difficilement comparables à ceux des femmes. La classification des trajectoires de tarifs donne quatre classes (voir figure 7 (b)). La classe $B$ représente 4644 patients ayant des séjours avec des tarifs relativement constants en début de parcours puis qui augmentent fortement au-delà de $4000 \mathrm{e}$ et diminuent ensuite. La classe $M$ 
caractérise 3894 patients ayant des séjours avec des tarifs oscillant autour de 2500 e. La classe $V$ rassemble 3595 patients avec des tarifs de séjours diminuant fortement au fur à mesure du parcours puis augmentant légèrement en fin de parcours. Pour finir, la classe $R$ concerne 2846 patients avec des tarifs de séjours de plus en plus élevés démarrant à moins de 4000 e pour finir à plus de $7000 \mathrm{e}$. Comme nous l'avons observé chez les femmes, la répartition des groupes de flux dans les classes de tarifs (figure 7 (c)) montre, dans le cas du groupe $I M$, qu'il y a une majorité de profils avec des tarifs élevés en début de parcours mais qui diminuent ensuite.

(a)

(b)

\begin{tabular}{cr|ccc|cccc}
\hline Groupe & & \multicolumn{4}{|c|}{ Classes de délais } & \multicolumn{4}{|c}{ Classes de tarifs } \\
Nom* & Effectif & $B$ & $M$ & $V$ & $B$ & $M$ & $V$ & $R$ \\
\hline Angine de poitrine & 4845 & 51 & 30 & 19 & 31 & 29 & 24 & 17 \\
Cardiopathie ischémique & 5422 & 49 & 29 & 22 & 31 & 33 & 22 & 14 \\
$I M$ & 4943 & 50 & 32 & 18 & 27 & 15 & 43 & 15 \\
\hline
\end{tabular}

*Le nom du groupe correspond au premier évènement du parcours de soins.

(c)

Figure 7. Profils de délais et de tarifs pour les hommes de +65 ans. Classes de (a) délais et (b) tarifs obtenues par kmlShape. (c) Répartition (en pourcentages) des groupes de flux dans les classes de délais et de tarifs ( $B, M, V$, R correspondent aux clusters des graphes (a) et (b)).

\section{Discussion}

\subsection{Flux de patients}

La visualisation des schémas de flux montre une différence des parcours entre genre avec : 1 ) des trajectoires plus longues chez les hommes ( .value $\left.<2,2.10^{-16}\right)$; 2) une diversification des étapes dans les schémas de flux plus importante chez les hommes. Ces observations s'expliquent par le fait que : 1) la population des hommes est plus nombreuse, ainsi, les possibilités d'observer des trajectoires différentes sont accrues ; 2) le taux de mortalité est plus élevé dans la population des femmes $(0,1 \%$ vs $0,08 \%$ pour les hommes). De plus, les femmes sont en moyenne significativement ( p.value $<2,2.10^{-16}$ ) plus âgées dans la même tranche d'âge (79 ans vs 75 ans pour les hommes). Ces deux derniers points peuvent expliquer des trajectoires plus courtes pour les femmes.

Nous avons identifié trois étapes clés dans les schémas de flux de patients : l'IM, l'Angine de poitrine et la Cardiopathie ischémique. La majorité des patients manifeste des signes de récidive de l'insuffisance coronarienne sous la forme d'une angine de poitrine. Beaucoup d'entre-eux font, par ailleurs, de nouveau un $I M$ et/ou développent une cardiopathie ischémique. D'autres sont concernés par des affections telles qu'un anévrisme, une ischémie, un trouble du rythme ou de la conduction, ou une insuffisance cardiaque. En outre, la part des décès représente environ $21 \%$ des hommes 
(resp. 32\% des femmes). Le décès se produit le plus souvent lors de la première hospitalisation. Nous retrouvons ici une partie des évolutions possibles de cette affection $(?, ?)$.

Les résultats obtenus par cette approche pourraient être intégrés dans un outil d'aide à la décision pour le clinicien. Il pourrait ainsi orienter ses recommandations et mettre en garde son patient sur les risques potentiels, en comparant son profil à celui de patients similaires. Ce type d'application est par exemple utilisée dans le cas de la prescription d'anti-thrombotiques afin d'éviter des risques d'interactions médicamenteuses (?, ?). Par ailleurs, cette méthode permet d'envisager une analyse territoriale. En effet, des études préalables ont mis en évidence un gradient Nord-Sud à la fois pour les hospitalisations, les réadmissions mais aussi pour la mortalité liée à l'IM (?, ?). L'exploration des flux en tenant compte de la région d'origine du patient en tant que paramètre contextuel offrirait la perspective comparative des flux soit en termes de soins, soit en termes d'évolution de la pathologie.

\subsection{Profils de délais et de tarifs}

Les profils de délais renseignent sur les occurrences des hospitalisations à venir, en lien avec la pathologie cardiaque. Aussi bien chez les hommes que chez les femmes, il y a une majorité de profils avec des délais de plus en plus courts et une proportion non-négligeable (environ 30\%) de profils avec, a contrario, des délais de plus en plus longs. Nos analyses permettent d'affirmer qu'à la suite d'un $I M$, les hospitalisations sont de plus en plus rapprochées dans le temps (avec une moyenne de trois à six mois selon le sexe) pour la majorité des patients. Ces résultats peuvent s'expliquer par le suivi de ces patients impliquant des contrôles réguliers, ou encore par une aggravation de leur état de santé nécessitant une ré-intervention. De plus, certaines techniques de revascularisation peuvent présenter des risques de ré-intervention supplémentaires. Par exemple, dans le cas de la pose d'endoprothèse, certains dispositifs médicaux sont susceptibles de provoquer des resténoses ${ }^{10}$ (?, ?). Toutefois, des investigations supplémentaires permettraient d'approfondir ces résultats.

L'évolution des tarifs de séjours est différente selon le sexe. Chez les hommes, les flux de patients ayant comme premier évènement une Angine de poitrine ou une Cardiopathie ischémique ont soit une évolution à la baisse, soit une forte évolution à la baisse, soit encore une tendance à la baisse avec un pic à 4200e en milieu de parcours. Par opposition, chez les femmes, ces flux ont, majoritairement, une évolution des tarifs à la hausse. Toutefois, les tarifs moyens n'excèdent pas $4000 \mathrm{e}$. En revanche, les flux de patients initialisés par un $I M$ ont, majoritairement, une évolution à la baisse de leurs tarifs avec un pic à près de 5000e (resp. 6000e) en moyenne chez les femmes (resp. pour les hommes) en début de parcours. Ainsi, si l'on compare ces résultats aux tarifs

10. Autrement dit, de ré-obstruction de l'artère coronaire. 
de référence ${ }^{11}$, on s'aperçoit que, quel que soit le profil de tarif, l'évolution moyenne des tarifs oscille dans une fourchette basse avec des tarifs inférieurs à $8000 \mathrm{e}$.

In fine, la synthèse de ces travaux soulèvent des interrogations au sujet des rythmes de ré-hospitalisation et notamment sur le lien entre le rythme des occurrences d'hospitalisation et l'évolution des tarifs de séjour. Par exemple, pour le flux initialisé par un $I M$, nous avons établi qu'il y avait à la fois une majorité de profils avec des hospitalisations rapprochées, mais aussi une majorité de profils avec des évolutions de tarifs à la baisse. Dans cet exemple, des investigations supplémentaires seraient nécessaires pour expliquer les raisons d'observer une réduction des délais dans les réadmissions à l'hôpital, mais aussi pour établir s'il y a un lien entre ces profils de délais et les profils de tarifs en diminution.

\subsection{Méthodes}

Dans le cadre de ce travail nous nous sommes focalisés sur un type de motifs spatio-temporels. Mais il en existe d'autres qui pourraient s'avérer utiles pour répondre à des questions médicales comme par exemple : quelles sont les trajectoires de soins qui conduisent à l'insuffisance cardiaque ? Ou encore quelles sont les évolutions d'une cardiopathie ischémique ? L'analyse des groupes convergents permettrait de répondre à la première question, tandis que l'analyse des groupes divergents permettraient de répondre à la deuxième (?, ?). En outre, l'approche pourrait être enrichie avec les diagnostics associés (DAS), les diagnostics reliés (DR) et les actes médicaux. Ainsi, au lieu du seul DP, le type d'hospitalisation serait décrit par une séquence non ordonnée d'itemsets diag, act), où diag serait l'itemset des diagnostics comprenant à la fois le DP, les DAS et les DR et act serait l'itemset des actes réalisés au cours du séjour hospitalier. Toutefois, la variabilité des situations étant importante, elle exige une étape préalable de simplification et de description des données pour que notre approche demeure efficace. Pour réaliser cette étape, une possibilité serait de procéder à la recherche des motifs maximaux $(?$, ?) dans ces séquences d'itemsets. Ensuite, une transformation des données telle que décrite dans $(?, ?)$ permettrait d'appliquer notre approche.

Par ailleurs, l'analyse des données intra-séjours offre d'autres perspectives en matière de planification au sein de l'établissement de soins. Dans ce domaine, des chercheurs ont développé un modèle, basé sur un arbre de régression, permettant de prédire les évènements ultérieurs pour un patient en cours d'hospitalisation (?, ?). Les auteurs se sont appuyés sur une représentation des parcours des patients au sein d'un même établissement de soins à l'aide de méthodes issues de la fouille de procédés (process mining). Cette dernière consiste à extraire de la connaissance à partir des évènements de trace d'exécution enregistrés dans les systèmes d'information (?, ?). La trajectoire

11. À titre de référence, le tarif d'une hospitalisation pour $I M$ varie entre 5600 e pour un niveau de sévérité faible à 23800 e pour un niveau de sévérité très élevé $(?, ?)$. 
d'un patient est la séquence de ses hospitalisations successives ou des évènements réalisés au cours de son séjour (actes, unités médicales fréquentés...). Autrement dit, c'est une séquence d'évènements où chaque évènement réfère à une étape bien définie (e.g. l'hospitalisation, la réalisation d'un acte), lié à un cas particulier (e.g. la maladie ayant conduit à l'hospitalisation). Par conséquent, il est envisageable d'appliquer ces méthodes telles que la découverte de procédés pour mettre en évidence les étapes clés de la trajectoire de soins (?, ?). Malgré certaines faiblesses, comme celles liées à la granularité des intervalles de temps empêchant le système d'identifier clairement la chronologie des évènements (?, ?), ce type d'approche a été utilisé dans différentes études avec des résultats encourageants notamment pour améliorer l'organisation des soins $(?, ?)$.

\subsection{Limites}

Nous pouvons formuler quelques remarques au regard de cette méthode :

- Le choix de la base de données : en effet, le PMSI est un outil d'allocation budgétaire, mais il a des limites dans le domaine épidémiologique (?, ?) car il expose à des imprécisions et à des erreurs tenant, entre autres raisons, à l'insuffisance de l'information, à des difficultés de codage et à des nomenclatures pouvant manquer de granularité (inadéquation de la codification de la maladie avec la réalité). Pourtant, elles représentent indéniablement une source importante d'informations. Elles couvrent la majorité des établissements de santé privés et publics sur le plan national et comprennent des données médicales sur tous les séjours hospitaliers. De nombreux chercheurs les ont utilisées : les travaux de $(?, ?)$ ont établi le réseau des établissements de santé de la région Lorraine ainsi que les interactions entre ces établissements ; les auteurs de $(?, ?)$ ont construit un modèle prédictif de la tendance des flux de patients au travers des différentes unités médicales ; enfin dans $(?, ?)$, les auteurs ont proposé un score mesurant le risque de décès consécutif à une intervention chirurgicale selon sa nature. Ces exemples concrets soulignent l'intérêt d'utiliser ces données pour la recherche médicale. Une alternative pour réduire le biais intrinsèque au codage pourrait être l'appariement des informations avec les bases de données du SNIIRAM (?, ?) (Système national d'information inter-régimes de l'assurance maladie).

- La comparaison à d'autres études : la littérature est riche de travaux sur les délais entre l'apparition des premiers symptômes et l'accès aux soins (?, ?), entre l'arrivée aux urgences et la prise en charge de l'IM (?, ?) mais aussi, des délais de réadmissions pour récidive (?, ?). À notre connaissance, il n'existe pas d'étude plus générale sur la ré-hospitalisation, qu'elle soit liée de près ou de loin à l'IM et à la variation des délais d'apparition de ces évènements. Par ailleurs, la plupart des auteurs $(?, ? ; ?)$ appuient leurs études de tarifs directs en tenant compte des frais d'hospitalisation et de la consommation de médicaments. L'accès aux bases du SNIIRAM (?, ?) permettrait de mener des analyses similaires.

- La durée d'observation : pour 33\% des patients le suivi a pu se faire sur cinq années, voire pratiquement six années pour ceux dont la première hospitalisation a été repérée en 2009. Mais pour bon nombre de patients (67\%), le suivi a été effectué entre 
moins d'un an à presque quatre ans. Ainsi, pour la majorité des patients le parcours est relativement court ne permettant pas d'avoir un recul suffisant pour observer tous les évènements clés du parcours de soins de ces patients. Par conséquent, les schémas de flux obtenus, représentés dans les diagrammes de Sankey (voir figure 5), ne font pas apparaître l'ensemble des parcours de soins possibles, car certains évènements ne sont pas suffisamment fréquents dans nos données pour ressortir lors de l'extraction de motifs. Certaines recherches analysant les tendances d'hospitalisation, de réadmission et de mortalité de l'IM peuvent avoir un recul allant jusqu'à 25 ans (?, ?; ?). L'accès aux données du PMSI ne nous permet pas à ce jour d'avoir un historique aussi important. En revanche, le recul des données du SNIIRAM est potentiellement plus long. Cependant cette base ne dispose pas du diagnostic de l'affection prise en charge mais seulement des soins afférents et des prescriptions médicamenteuses.

\section{Conclusion}

Dans cet article nous avons présenté une méthode novatrice de caractérisation des flux de patients, ayant présenté un $I M$, à partir des données du PMSI. Cette méthode s'articule en deux grandes phases. Tout d'abord, nous avons recherché des motifs spatio-temporels dans les trajectoires des patients. L'originalité de l'approche est qu'ici la spatialité a été assimilée à celle de proximité de pathologies (?, ?). De plus, l'aspect temporel est lié à la survenue d'une hospitalisation. Ensuite, nous avons classé leurs trajectoires de durées et de tarifs afin de déterminer des profils types en termes d'occurrence et de facturation de ces évènements.

La visualisation des schémas de flux renseigne sur les évolutions de la pathologie cardiaque. Nous avons retrouvé des éléments connus du domaine de la cardiologie. Par ailleurs, nous avons pu établir que dans le cas des parcours débutant par un $I M$, la majorité des occurrences suivantes sont de plus en plus proches dans le temps avec une tendance de l'évolution des tarifs à la baisse de ces évènements. Néanmoins, un tiers de cette population est concerné par une augmentation des tarifs, ou des occurrences d'hospitalisations de plus en plus éloignées dans le temps.

Ce type d'approche peut apporter une aide à la prise en charge en prévoyant, par exemple, un parcours de soins avec des visites programmées comme cela a été fait dans le cas du cancer (?, ?). Elle peut également être à la base d'analyses comparatives des parcours de soins pour mieux comprendre les résultats observés, par exemple dans le cas du flux initialisé par un $I M$. Ceci pourrait par ailleurs induire des choix stratégiques de soins et favoriser la réduction des coûts tout en maintenant la qualité des soins $(?, ?)$. Pour étendre ces travaux, nous envisageons de prendre en compte la coévolution des trajectoires de délais et de tarifs avec la méthode proposée par (?, ?) afin d'établir si elles sont liées. 


\section{Bibliographie}

\section{Les auteurs}

Jessica Pinaire est data scientist dans l'équipe de recherche EA2415 depuis 2018. Elle travaille sur les données de santé depuis plus 2012. Ses recherches l'ont conduite à se focaliser sur la thématique des trajectoires de patients combinant des technique de fouille de données avec celles de biostatistiques. Elle est titulaire d'un doctorat de biostatistiques de l'université de Montpellier.

Jérôme Azé Professeur des Universités à l’Université de Montpellier depuis 2013. Il effectue ses recherches au LIRMM. Il est spécialisé dans la fouille de données avec des applications particulières aux données de santé avec l'objectif de leur donner une plus-value médicale. Il est l'auteur de plus de 80 articles dont les plus récents sont centrés sur les thématiques de la fouille de données appliqués à des problématiques médicales.

Sandra Bringay Professeur des Universités Paul Valéry Montpellier depuis 2016. Elle effectue ses recherches au LIRMM. Elle est spécialisée dans la fouille de données avec des applications particulières aux données de santé avec l'objectif de leur donner une plus-value médicale. Elle est l'auteur de plus de 90 articles dont les plus récents sont centrés sur les thématiques de la fouille de données appliqués à des problématiques médicales

Pascal Poncelet est professeur à l'Université de Montpellier et responsable du projet Advanse (Advanse Analytics for Data Science) au sein du Laboratoire d'Informatique, de Robotique et de Microélectronique de Montpellier (LIRMM). Ses domaines de recherches concernent les approches avancées d'analyses de données (visualisation analytique, apprentissage automatique) pour les applications émergentes et la fouille de données. Il est titulaire d'un doctorat en informatique de l'Université de Nice-Sophia Antipolis.

Christophe Genolini a passé 15 ans dans la recherche publique, il est maintenant start-uppeur. Il crée $\mathrm{R}++$, un logiciel d'analyse statistique haute performance, jusqu'à 800 fois plus rapide que les logiciels classiques et surtout intégré dans une Interface Homme Machine simple et intuitive.

Paul Landais est professeur émérite de l'Université de Montpellier. Docteur en Santé Publique, Néphrologue, Epidémiologiste clinicien, il dirige l'UPRES EA2415 : Aide à la Décision pour une Médecine Personnalisée, Institut Universitaire de Recherche Clinique depuis 2012. Il est également le co-coordonnateur du programme national RaDiCo (Rare Disease Cohorts) Inserm U933 Paris Hôpital Trousseau. Il est l'auteur de 260 publications dans des revues internationales. 
